\title{
VIOLÊNCIA SEXUAL NO NAMORO: OS ATLETAS UNIVERSITÁRIOS COMO GRUPO DE RISCO?
}

\author{
Judite Peixoto ${ }^{1}$ \\ Marlene Matos ${ }^{1}$ \\ Carla Machado ${ }^{1}$
}

[Enviado a 04-09-2012. Aceite a 05-01-2013]

Resumo: Estudos internacionais realizados em contexto universitário apontam consistentemente para a sobrerrepresentação dos atletas como perpetradores frequentes de agressões sexuais nas relações íntimas. A literatura científica tem vindo a sustentar que há aspetos da cultura atlética que contribuem para a maior adesão a mitos socioculturais e a atitudes estereotipadas de género, aumentando a propensão dos atletas para a adoção de condutas sexualmente abusivas. Este estudo exploratório procurou analisar as possíveis diferenças entre duas amostras independentes de estudantes universitários do sexo masculino - atletas e não atletas -, a dois níveis: 1) grau de tolerância/aceitação face à violência sexual sobre as mulheres; 2) taxa de prevalência e tipologia de comportamentos sexualmente violentos perpetrados no contexto das suas relações amorosas não conjugais (namoro). Para a recolha de dados foram utilizados a Escala de Crenças sobre a Violação (ECV) e o Sexual Experiences Survey - Short-Form Perpetration (SES-SFP), que foram administrados a 50 atletas - praticantes de modalidades desportivas de competição de contacto -, e 50 não atletas universitários. Os resultados sugerem que os atletas não apresentam níveis superiores de legitimação da violência sexual contra as mulheres, nem evidenciam uma taxa superior de perpetração de atos de violência sexual na intimidade face ao grupo dos não atletas. No plano dos comportamentos, ambos os grupos apresentam taxas preocupantes de perpetração de atos sexuais severos na intimidade (e.g., violação).

Palavras-chave: violência sexual, namoro, atletas universitários, crenças e comportamentos.

Sexual violence in dating relationships: Are college athletes at higher-risk? (Abstract): International studies developed at the university context consistently point

\footnotetext{
${ }^{1}$ Escola de Psicologia, Universidade do Minho 
out for the over-representation of athletes as frequent perpetrators of sexual assault in intimate relationships. The scientific literature has argued that there are aspects of the athletic culture that contribute to greater adherence to sociocultural myths and stereotypical attitudes to gender, increasing the propensity of athletes to commit more sexually abusive behaviors. This exploratory study sought to analyze the possible differences between two independent samples of male college students - athletes and non-athletes - at two levels: 1) degree of acceptance of sexual violence against women, 2) prevalence rate and types of sexually violent behaviors perpetrated in non-marital romantic relationships (dating). For the data collection we used the Beliefs Scale about Rape (ECV) and the Sexual Experiences Survey - Short Form-perpetration (SES-SFP), which were both administered to 50 college athletes of contact sports and to 50 non-athletes. The results suggest that college athletes do not report higher overall levels of legitimacy of sexual violence against women, and that they do not have higher rates of committing acts of sexual violence in the intimacy. In terms of behavior, there is empirical evidence that both college athletes, as non-athletes, report disturbing perpetration levels of severe acts of sexual assault in private (e.g., rape).

Keywords: sexual violence, dating, college athletes, beliefs and behaviors.

\section{Introdução}

A violência sexual nas relações de namoro tem vindo a ser, progressivamente, reconhecida como um dos problemas sociais mais significativos que a sociedade atual enfrenta.

O progressivo investimento da comunidade científica internacional no estudo deste fenómeno, sobretudo a partir da década de 80 , tem vindo a comprovar que esta é uma forma de abuso predominantemente assimétrico entre sexos, com uma maior vulnerabilidade das mulheres para a vitimação sexual e dos homens para a perpetração de atos sexualmente abusivos (e.g., Jackson, 1999; Mahoney, Williams, \& West, 2001; Schwartz \& DeKeseredy, 1997). Trata-se ainda de uma problemática com indicadores de prevalência cada vez mais preocupantes (Banyard, Plante, \& Moynihan, 2004).

A investigação empírica sobre este tema (e.g., DeKesereday \& Schwartz, 1998; Carr \& VanDeusen, 2004) tem vindo a centrar-se no contexto universitário, demonstrando que os estudantes universitários se assumem como um forte grupo de risco para a ocorrência de violência sexual, tanto ao nível da vitimação, como da perpetração, comparativamente com a população em geral. A literatura da especialidade sustenta que, entre este grupo populacional, o género masculino constitui o principal fator de risco para a perpetração da violência sexual (Malamuth, Heavey, \& Linz, 1996; McMahon, 2000) e que, na grande maioria dos casos, esta é cometida por um 
conhecido da vítima, frequentemente pelo parceiro amoroso (e.g. Gross, Winslett, Roberts, \& Grohn, 2006; Himelein, 1995).

Mais recentemente, há evidência empírica a reportar a existência de subculturas sexistas ou grupos de risco específicos entre a população universitária para a perpetração de atos de violência sexual sobre as mulheres, como as associações estudantis e os atletas universitários (McMahon, 2007), pela cultura da hipermasculinidade que veiculam e pelos modelos legitimadores de comportamentos sexualmente coercivos que promovem (Rozee \& Koss, 2001). Assim, parece haver determinados aspetos da cultura atlética que desempenham um papel significativo na propensão quer para a adesão a perigosos mitos socioculturais, legitimadores da violência sexual contra as mulheres, quer para o cometimento, mais frequente, de atos de violência sexual na intimidade (McMahon, 2004; McMahon, 2007; Sanday, 1990).

Assim sendo, e sobretudo devido ao facto da violência sexual (i.e., coerção sexual, tentativa de violação e violação sexual), perpetrada por atletas universitários no contexto das relações de namoro, ser ainda um tema omisso no discurso científico nacional, procurámos, através de um estudo exploratório, produzir conhecimento sobre as crenças que estes estudantes universitários, do sexo masculino, praticantes de modalidades desportivas de competição de contacto, possuem relativamente à violência sexual contra as mulheres na intimidade e determinar a prevalência e tipologia de atos sexualmente abusivos praticados. Deste modo, procurámos perceber de que forma o grau de adesão aos mitos socioculturais se relaciona com a adoção de práticas sexuais abusivas, no contexto das relações amorosas.

\section{Enquadramento teórico}

\section{Delimitações terminológicas e tipologias de violência sexual}

Várias definições são preconizadas na literatura sociológica e psicológica sobre a violência sexual, todas elas enfatizando a noção de conduta sexual não consentida (Schwartz \& DeKeseredy, 1997). Num sentido lato, considera-se que a violência sexual integra "qualquer tipo de relação sexual sem consentimento, desde a agressão sexual, a violação sexual, o controlo sexual dos direitos reprodutores e outras formas de manipulação sexual, utilizadas pelos perpetradores com a intenção de causar perturbação emocional, sexual e fisica na outra pessoa" (Abraham, 1999, p. 592, citado por Caridade \& Machado, 2008). Num sentido particular, a violência sexual nas relações de namoro pode ser entendida como "a perpetração ou ameaça de um ato de violência de natureza sexual, pelo menos por um membro do casal de namorados sobre o outro, no contexto de uma relação íntima. Esta violência contempla qualquer forma de agressão sexual, variando do simples 
contacto sexual indesejado à situação extrema de violação sexual, com a intenção de obter controlo, poder e autoridade sobre a vítima" (adap. Center Disease Control, 2000, citado por Castro, Cruz, \& Martins, 2005). Neste sentido, a violência sexual tem vindo a ser conceptualizada como um contínuo entre diferentes tipos de comportamentos sexualmente violentos (Koss \& Oros, 1982): (1) o contacto sexual (e.g., carícias e beijos), geralmente alcançado através do recurso à pressão verbal, ao abuso de autoridade ou à utilização de ameaças ou da força física; (2) a coerção sexual, que inclui praticar relações sexuais indesejadas, com recurso privilegiado à ameaça através da coerção verbal ou do abuso de autoridade; (3) a tentativa de violação, que implica tentar ter relações sexuais indesejadas, através do uso da ameaça, da força ou de drogas e/ou álcool; (4) a violação sexual, que inclui a prática de relações sexuais indesejadas e implica a utilização da ameaça ou uso da força física ou, ainda, o uso de drogas e álcool, podendo englobar vários tipos de atos sexuais não consentidos (relações sexuais oral, anal, penetração digital ou com recurso a objetos).

\section{Indicadores de prevalência}

A revisão dos dados epidemiológicos da produção científica internacional sobre a violência sexual na intimidade revela que, apesar da crescente expansão dos estudos sobre o tema, há vários obstáculos à recolha de dados de prevalência sobre esta problemática, nomeadamente a grande variabilidade nas opções metodológicas utilizadas (e.g., design dos estudos, tipo de amostragem, tipo(s) de abuso avaliado(s), instrumentos de recolha de dados administrados) (Caridade \& Machado, 2010). Concomitantemente, há autores (e.g., Caridade \& Machado, 2010; Martins \& Machado 2010) que sugerem a ambiguidade concetual associada à definição e operacionalização de atos sexuais abusivos ou violentos, a centração predominante na medição do abuso físico (Gover, 2004) e a ausência de estudos longitudinais (Glass, Fredland, Campbell, Yonas, Sharps \& Kub, 2003) como importantes obstáculos ao conhecimento mais preciso destes indicadores.

Determinar o número de ofensores e vítimas de atos de violência sexual ocorridos na intimidade implica ainda ter presente que muitos de comportamentos abusivos não são relatados pelo facto de muitas vítimas e perpetradores não perceberem o sexo coercivo como uma experiência de violência sexual, sobretudo quando o contexto de ocorrência é uma relação amorosa (Humphrey \& White, 2000). Assim, o fracasso em se entender a violência sexual no namoro como uma transgressão grave e séria pode ajudar a perceber, por exemplo, porque é que, de acordo com as estimativas, só 3\% das violações nos EUA resultam em processos judiciais (Truman, Tokar, \& 
Fisher, 1996). A nível nacional, estima-se que apenas $1 / 5$ das violações sejam participadas às autoridades (Costa \& Alves, 1999).

Apesar destes obstáculos à recolha de dados epidemiológicos sobre violência sexual na intimidade, a literatura científica é consistente na ideia de que a agressão sexual distingue-se doutras formas de violência por haver uma clara propensão ou vulnerabilidade da mulher para a vitimização sexual comparativamente com os homens, que se assumem mais frequentemente como perpetradores (e.g., Jackson, 1999; Mahoney et al., 2001; Schwartz \& DeKeseredy, 1997). Neste sentido, Molidor, Tolman e Kober (2000) encontraram diferenças significativas de género ao nível da vitimação sexual, em que cerca de $18 \%$ das mulheres referem terem sido vitimadas sexualmente, comparativamente com $0,3 \%$ dos homens. Concomitantemente, a investigação aponta para que a maioria das agressões sexuais ocorra em contextos relacionais de intimidade, nomeadamente em relações de namoro (Koss, Dinero, Seibel, \& Cox, 1988), não se circunscrevendo às relações conjugais (Price, Beyers, \& Dating Violence Research, 1999). De facto, estima-se que, nos EUA, a agressão sexual entre namorados ou conhecidos, comummente designada na literatura internacional como date or acquaintance rape, tem aumentado dramaticamente nos últimos 30 anos (Day, 1994). A taxa de prevalência do date or acquaintance rape situa-se entre os 80 e os $90 \%$ de todas as violações, enquanto o stranger rape apenas ocorre 10 a $20 \%$ das vezes (Koss, et al., 1988; Yeater \& O'Donohue, 1999). De facto, a investigação científica tem demonstrado consistentemente que há mais mulheres violadas pelos próprios namorados ou conhecidos do que por estranhos (Russell, 1984, citado por Yeater \& O’Donohue, 1999). O estudo de Koss, Gidycz e Wisniewski (1987) foi pioneiro e significativo nos resultados que obteve: $95 \%$ das violações envolvem um único agressor; $84 \%$ das vítimas conhecem o perpetrador; $57 \%$ dos violadores são os próprios namorados. Outros autores (e.g., Jackson \& Davis, 2000) estimam ainda que uma em cinco mulheres já foi alvo de algum tipo de vitimação sexual, durante a sua história de namoro.

Vários estudos e inquéritos internacionais, realizados em campi universitários (e.g., Day, 1994; Koss \& Oros, 1982; Muehlenhard \& Linton, 1987), revelaram igualmente taxas alarmantes de agressão sexual e violações em situações de namoro entre a população universitária, estimando que aproximadamente $50 \%$ das estudantes universitárias tenham experienciado alguma atividade sexual indesejada nos seus relacionamentos amorosos.

Por sua vez, os estudos sobre a perpetração sexual masculina desenvolvidos em contexto universitário (e.g., Abbey, McAuslan, Zawacki, Clinton, \& Buck, 2001; Muehlenhard \& Linton, 1987) apontam para indicadores de agressão sexual entre os $25 \%$ e os $75 \%$. No estudo de Koss, Gidycz e Wisniewski (1987), 25\% dos homens universitários relataram terem estado envolvidos em algum tipo de agressão sexual. Num outro estudo mais recen- 
te (Loh, Gidycz, Lobo, \& Luthra, 2005), realizado com estudantes do sexo masculino, $31 \%$ dos participantes admitiram a prática de comportamentos sexualmente agressivos sendo que destes, $27,5 \%$ reconheceu ter exercido violência sexual moderada e 3,7\% violência sexual severa. Em suma, os estudos realizados em campi universitários, revelam não só que os homens universitários são perpetradores frequentes deste tipo de vitimação, mas também que as estudantes universitárias são vítimas frequentes de agressão sexual no contexto das suas relações amorosas.

Embora parcos, os estudos nacionais realizados em contexto universitário a propósito da problemática da violência na intimidade juvenil obtiveram resultados interessantes, sendo que alguns deles corroboram as evidências empíricas internacionais. De salientar a investigação levada a cabo por Paiva e Figueiredo (2004) sobre o abuso na intimidade dos jovens adultos portugueses, onde a coerção sexual foi o segundo tipo de abuso mais prevalente na amostra, com uma taxa de perpetração e vitimação de $18,9 \%$ e $25,6 \%$, respetivamente. No estudo de Costa e Sani (no prelo) sobre a violência nas relações amorosas dos estudantes universitários, a taxa de vitimação sexual (i.e., coerção sexual) situava-se nos $24,4 \%$ e a de perpetração nos 19,8\%. Num outro estudo (Machado, Matos, \& Moreira, 2003), os indicadores de vitimação associada à coerção sexual situavam-se nos $1,3 \%$ e os de perpetração nos $0,3 \%$.

Em suma, apesar da proliferação de estudos nesta área, a disparidade observada entre os dados epidemiológicos recolhidos corrobora a "necessidade de uniformização dos critérios concetuais e metodológicos" subjacentes à produção científica na área da violência na intimidade juvenil (Caridade \& Machado, 2010, p. 22).

Fatores de risco para a violência sexual no namoro: A relevância dos fatores socioculturais

Uma revisão da literatura sociológica sobre as variáveis etiológicas implicadas no comportamento sexualmente agressivo permite sinalizar uma complexa rede de fatores multidimensionais: socioculturais (e.g., os valores culturais e os guiões do papel de género tradicional), institucionais (e.g., grupos de pares, escolas), interpessoais (características relacionais, características da vítima, mal-entendidos entre vítima e ofensor) e individuais (e.g., atitudes, traços de personalidade, diferenciais de género) que, de uma forma mais distal ou proximal, favorecem a ocorrência da violência sexual na intimidade (Davis, 2000).

A este nível, o modelo integrativo de Berkowitz (1992) surge como uma compilação de múltiplos fatores de socialização, personalidade, características situacionais e mitos socioculturais de perpetradores e vítimas, inte- 
grados na conceptualização dos fatores de risco específicos associados à violência sexual contra as mulheres. O pressuposto básico deste modelo teórico é que as atitudes e crenças que toleram a violência sexual criam o potencial para que atos sexualmente violentos possam ocorrer, sendo ainda reforçados por fatores pessoais e experiências sexuais prévias, designadamente o processo de socialização dos papéis do género tradicionais; o uso e/ou abuso de álcool; as características de personalidade (e.g., externalização da culpa, impulsividade, agressividade, hostilidade e falta de empatia); e o abuso e negligência infantil (Carr \& VanDeusen, 2004).

As teorias feministas deram, igualmente, um importante contributo para a compreensão da violência sexual, considerando que as crenças e atitudes socioculturais que legitimam a violência sexual contra as mulheres são largamente responsáveis pela sexualidade coerciva dos homens e pela grande tolerância social face à violência no contexto das relações íntimas (Brownmiller, 1975; Rozée, 1993).

Em suma, várias perspetivas teóricas explicativas da agressão sexual, designadamente as abordagens feministas e as teorias socioculturais, defendem que os papéis estereotipados de género e os guiões sociais que orientam o comportamento de homens e mulheres no contexto interpessoal (e.g., "os homens são dominadores e agressivos" e "as mulheres são passivas e submissas") potenciam a criação de mitos socioculturais (e.g., "As mulheres dizem não a uma relação sexual quando, na verdade, querem dizer sim") e outras atitudes sexistas que facilitam a perpetração de atos de violência sexual contra as mulheres (ibidem). Conceptualizadas como estereótipos ou falsas crenças sobre a violação, (Burt, 1980), este tipo de crenças visa, assim, negar ou justificar a agressão sexual masculina (Lonsway \& Fitzgerald, 1994).

Paralelamente, do ponto de vista empírico, um corpo extenso de investigação tem demonstrado consistentemente associações significativas entre a aceitação de mitos socioculturais com atitudes sexistas e comportamentos sexualmente violentos (Lonsway \& Fitzgerald, 1994). Neste sentido, um pressuposto básico relativamente à violência sexual parece ser pois o de que quanto maior for a adesão às noções e ideais socioculturais de masculinidade, maior será a tendência para vitimar sexualmente as mulheres, especialmente no contexto das relações íntimas. A aceitação dos mitos sobre a violação sexual surge, assim, consistentemente referenciada nos estudos empíricos como estando associada ao comportamento sexualmente agressivo (Lonsway \& Fitzgerald, 1994). Em particular, os estudos realizados com estudantes universitários (e.g., Koss \& Cleveland, 1997; Sanday, 1990) sugerem que estes poderão estar expostos a uma cultura agressiva e hipermasculinizada que encoraja atitudes e práticas de dominância dos homens e objetificação das mulheres (Martins \& Machado, 2010). Neste contexto, a pertença a um grupo de pares (e.g., associações de estudantes, equipas des- 
portivas de modalidades de contacto) pode desempenhar um papel determinante ao nível da perpetração de atos sexualmente agressivos (e.g., Malamuth, Sockloskie, Koss, \& Tanaka, 1991).

Adicionalmente, há estudos que sugerem traços de personalidade, como a hostilidade masculina (e.g., Hunter et al., 2004; Mosher \& Anderson, 1986, citados por Truman et al., 1996), enquanto preditores significativos de perpetração sexual. Assim, conceptualiza-se que os homens que evidenciam níveis elevados de hostilidade tendem a adotar determinados estereótipos e mitos sexuais e, deste modo, legitimam mais facilmente a violência interpessoal. Neste contexto, o estudo de Truman, Tokar e Fisher (1996) procurou compreender a relação entre o papel do género masculino e as atitudes que suportam a violência sexual no namoro. Para tal, focou-se na análise de constructos relacionados com a masculinidade, constatando que as atitudes contra as mulheres emergem como o preditor mais consistente das atitudes e crenças legitimadoras da violação nas relações amorosas.

Adicionalmente, a investigação aponta para que a frequência das relações amorosas e o número de parceiros sexuais, as diferenças de poder entre homem e mulher, as atitudes adversas sobre os relacionamentos amorosos, a aceitação dos papéis de género tradicionais, os locais de encontro e o uso de álcool e/ou drogas constituam alguns dos mais significativos fatores de risco proximais para que a agressão sexual ocorra no contexto de uma relação amorosa (Koss \& Dinero, 1989; Muehlenhard \& Linton, 1987). Assim, conclui-se que os indivíduos que namoram mais frequentemente e/ou possuem mais parceiros amorosos têm uma maior probabilidade de experienciar e/ou cometer violência sexual, em virtude de uma maior oportunidade para tal (Koss \& Dinero, 1989). Outros estudos (e.g., Muehlenhard \& Linton, 1987) sugerem como fatores de risco as diferenças de idade e certos comportamentos (como, por exemplo, ser o homem a iniciar uma relação de namoro, a pagar as despesas e a conduzir) aumentam a probabilidade de vitimação sexual.

Há, igualmente, evidência empírica (e.g., Miller \& Marshall, 1987; Mynatt \& Allgeier, 1990, citados por Söchting, Fairbrother, \& Koch, 2004) que aponta para que a probabilidade de uma agressão sexual seja tanto maior quanto mais isolado e privado for o local de encontro dos namorados. $\mathrm{O}$ estudo de Muehlenhard e Linton (1987) encontrou uma percentagem de 81\% de ataques sexuais ocorridos em carros e locais privados (e.g., apartamento ou casa do namorado). Assim, os homens tendem a interpretar o à-vontade da mulher para ir a certos locais como sinal de que esta está interessada e disponível para o envolvimento sexual (Muehlenhard \& Linton, 1987). Frequentar locais ermos parece aumentar igualmente a ocorrência de atos de agressão sexual porque há uma menor probabilidade de interrupção por outros (ibidem). 
Por sua vez, o uso/abuso de álcool parece ser um fator situacional interveniente em qualquer tipo de agressão sexual. Estima-se que cerca de 40 a $65 \%$ das mulheres que sofreram algum tipo de violência sexual tenham consumido álcool antes da agressão (Frintner \& Rubinson, 1993; Harrington \& Leitenberg, 1994; Miller \& Marshall, 1987; Wilson \& Durrenberger, 1982, citados por Söchting et al., 2004). A revisão de literatura sobre este tema sugere que o álcool interfere de forma significativa com a capacidade para consentir o envolvimento sexual, detetar o perigo, bem com a capacidade para resistir mais prontamente a uma agressão sexual (Söchting et al., 2004). Os mal-entendidos entre homens e mulheres, como a sexualização do comportamento da mulher, também parecem dar azo a um aumento da probabilidade de contatos sexuais indesejados entre parceiros amorosos (Muehlenhard \& Linton, 1987). Assim, não raro, um qualquer comportamento, descomprometido de intenções sexuais (e.g., abraço, beijo), pode ser interpretado como um preliminar para a relação sexual.

Atletas universitários e violência sexual contra as mulheres no namoro: Das crenças aos comportamentos

Nos últimos anos tem aumentado o interesse da literatura científica pelo papel dos atletas universitários na violência sexual contra as mulheres, apesar dos estudos neste domínio serem ainda escassos. Não obstante, tem-se assistido a um grande debate sobre se os atletas têm, de facto, maior probabilidade para cometerem atos de violência sexual ou se estamos simplesmente perante uma publicidade especulativa em torno do seu estatuto e da sua visibilidade social (McMahon, 2007).

A investigação sobre a violência sexual na população universitária norte-americana tem dado particular atenção a duas formas frequentes de agressões sexuais, em que os atletas universitários se assumem como os principais perpetradores: o gang rape e o date or acquaintance rape (Day, 1994).

A violência sexual perpetrada por um gang é uma realidade frequente nos campus universitários norte-americanos, sobretudo entre pessoas que se conhecem (Gordon \& Riger, 1989 citado por Day, 1994). Esta forma de agressão sexual ocorre mais frequentemente entre grupos de homens que foram socializados em torno do consumo de álcool, da pressão de pares e da objetificação da mulher. As associações de estudantes e as equipas de atletas universitárias são, neste contexto, os perpetradores primários de atos de violência sexual (Day, 1994). Enhard e Sandler (1981, citados por Day, 1994) levaram a cabo um estudo sobre 50 casos registados de gang rape e descobriram que $30 \%$ destes casos envolviam atletas universitários.

Não obstante, os investigadores têm caracterizado a maioria dos ataques sexuais envolvendo a população universitária como sendo do tipo date or acquaintance rape, ou seja, comummente perpetrados por uma pessoa 
que as vítimas conhecem (Day, 1994). Este tipo de ataques envolve, geralmente, o uso de álcool ou outras drogas ilícitas e, frequentemente, ocorre num contexto de intimidade entre vítima e agressor (Warshaw, 1988, citado por Day, 1994). O álcool parece ser, de facto, uma das variáveis situacionais explicativas das elevadas taxas de violência sexual nas equipas atléticas e nas associações de estudantes (Koss \& Gaines, 1993). Também neste tipo de violação há evidência empírica substancial a documentar que os atletas universitários se assumem como um grupo de alto-risco para a perpetração de violência sexual, quando comparados com não atletas (Day, 1994).

De facto, há um corpo de estudos empíricos que, embora parcos, concluíram, consistentemente, que os atletas estão sobrerrepresentados como perpetradores frequentes de ataques sexuais entre a população universitária e que há aspetos da cultura atlética que suportam a evidência de maior aceitação e perpetração de atos de violência sexual (e.g., Boeringer, 1996; Boeringer, 1999; Crosset, Benedict, \& McDonald, 1995; Crosset, Ptacek, McDonald, \& Benedict, 1996; Frintner \& Rubinson, 1987; Koss \& Gaines, 1993). No estudo de Crosset, Ptacek, McDonald e Benedict (1996), apesar dos atletas-estudantes da I Divisão constituírem apenas 3,3\% do corpo estudantil, eles representavam $19 \%$ dos perpetradores de ataques sexuais. Por sua vez, Fritner e Rubinson (1993) obtiveram resultados ainda mais surpreendentes. No seu estudo, apesar de os atletas constituírem menos de $2 \%$ do total dos estudantes universitários, eles representavam cerca de 22,6\% dos perpetradores de relações vaginais, orais ou anais forçadas. Estes resultados significam que, apesar da pequena representação que os atletas têm ao nível da população académica, este grupo está sobrerrepresentado em termos daqueles que relatam mais frequentemente tentativas ou consumação de atos de violência sexual, abuso sexual, agressão física ou intimidação (Schwartz \& DeKeseredy, 1997). Outros estudos, incluindo um do National Institute of Mental Health (1990), documentam que os atletas participam em 1/3 de todos os ataques sexuais que ocorrem no contexto universitário, nos EUA (Melnick, 1992; Schwartz \& DeKeseredy, 1997).

Neste sentido, alguns autores (e.g., Ehrhart e Sandler, 1992; Sanday, 1981; Sanday, 1990; O'Sullivan, 1991, citados por Crosset et al., 1995) sustentam que a pertença a grupos onde a segregação sexual existe (e.g., equipas atléticas, forças armadas, associações de estudantes) favorece a prática de atos sexuais abusivos. Adicionalmente, a afiliação a desportos agressivos e competitivos, que envolvem o contacto físico direto com os adversários ${ }^{2}$, parece correlacionar-se positivamente com taxas mais elevadas de perpetra-

\footnotetext{
2 Designados na literatura internacional como contact sports (e.g., futebol, hóquei, basquetebol), por oposição aos non-contact sports, aqueles desportos em que não há o contacto físico direto com os adversários (e.g., voleibol, natação).
} 
ção de ataques sexuais nas relações íntimas. A este nível, Kemler (1988, citado por Smith \& Stewart, 2003) encontrou, no seu estudo, níveis mais elevados de agressão reativa nos atletas de desportos de contacto do que nos atletas de desportos de não-contacto. Por sua vez, Brown e Davis (1978, citados por Smith \& Stewart, 2003) obtiveram indicadores de maior legitimação da violência entre os atletas dos contact sports do que nos não atletas. Há autores que afirmam que os atletas de modalidades desportivas de contacto tendem a apresentar níveis elevados de competitividade, de orientação para ganhar e taxas mais elevadas de agressão sexual, assim como atitudes legitimadoras da violação e maiores níveis de hostilidade em relação às mulheres. Bredemeier, Shields, Weiss e Cooper (1984, citados por Smith \&Stewart, 2003) aditam que a participação dos homens em modalidades desportivas de contacto apresenta uma correlação positiva com o desenvolvimento de atitudes e tendências agressivas. Assim, há uma clara evidência da associação entre altos níveis de agressão e a maior legitimação da violência nos atletas de desportos de contacto do que nos atletas de desportos onde não há contacto ou em não atletas.

Os estudos com estes agressores são unânimes em considerar que estes homens são socializados num ambiente de masculinidade hegemónica (Connell, 2005) e de hipermasculinidade (Day, 1994) que veiculam, entre outros valores, controlo, força física, proeza sexual, submissão da mulher, competitividade, invulnerabilidade, homofobia e consumo de álcool, o que poderá ter implicações ao nível dos seus relacionamentos amorosos (Forbes, Adams-Curtis, Pakalka, \& White, 2006). Neste sentido, Russell (1983, citado por Schwatz \& DeKeseredy, 1997) defende que o trabalho exaustivo para tornar os atletas dominantes, agressivos e competitivos dentro de campo pode levar a um aumento destes comportamentos fora dele, nomeadamente no contexto das relações amorosas.

\section{Estudo empírico}

\section{Objetivos}

Este estudo transversal, de carácter exploratório, tem como objetivo primordial perceber se os estudantes universitários do sexo masculino, praticantes de modalidades desportivas de competição de contacto, designados neste estudo por atletas, por um lado, evidenciam um nível superior de legitimação/tolerância face à violência sexual sobre as mulheres e, por outro, se apresentam uma taxa de prevalência de atos de violência sexual no namoro mais elevada, quando comparados com um grupo de não atletas. Mais especificamente, pretendemos: a) descrever as crenças dos atletas e dos não atletas em relação à violência sexual contra as suas parceiras amorosas, através da 
identificação do grau de tolerância/legitimação em relação a esta problemática e a tipologia de crenças que concorrem para a sua legitimação; b) recolher dados sobre a prevalência e frequência de perpetração de diferentes tipos de violência sexual (i.e., coerção sexual, tentativa de violação e violação) nas relações de namoro passadas e presentes dos atletas e dos não atletas; c) estabelecer e analisar as possíveis diferenças entre atletas e não atletas, em termos de crenças e comportamentos de violência sexual nas suas relações amorosas.

\section{Método}

\section{Amostragem}

Trata-se de uma amostra de conveniência, constituída por 100 estudantes universitários do sexo masculino. Estes participantes integraram dois grupos independentes de estudantes universitários, semelhantes em termos de características sociodemográficas (sexo e idade) e formativas (tipo de curso e ano académico). $\mathrm{O}$ grupo de atletas foi constituído por 50 participantes, praticantes de cinco modalidades desportivas de competição, na vertente desportos de contacto, designadamente: 14 praticantes de futsal, 11 de basquetebol, 13 de andebol, 6 de pólo aquático e 6 de judo. Neste grupo, as idades variam entre os 18 e os 38 anos, sendo a média 22,9 e o desvio-padrão 3,61. A média de anos de formação era $1,2(D P=0,39)$ e a maioria dos atletas $(n=41,82 \%$,) frequentava cursos do domínio das Ciências Exatas, sendo que apenas 9 atletas (18\%) frequentavam cursos do domínio das Ciências Humanas e Sociais. O grupo de não atletas foi composto por 50 sujeitos, com idades compreendidas entre os 19 e os 28 anos, sendo a média 22,3 e o desvio-padrão 2,73. A média de ano de formação era 2,9 $(D P=$ $1,42)$ e a maioria dos não-atletas $(n=41,82 \%$,) frequentava cursos do domínio das Ciências Exatas, sendo que apenas 9 atletas (18\%) frequentavam cursos do domínio das Ciências Humanas e Sociais. A aplicação do teste não paramétrico Mann-Whitney demonstrou que não há diferenças estatisticamente significativas entre atletas e não atletas ao nível da idade $(U=1145.5$, $p=.468)$ e do ano académico $(U=1168, p=.563)$.

\section{Instrumentos}

Os instrumentos utilizados para a recolha de dados foram a Escala de Crenças sobre a Violação (ECV), um questionário de autorrelato, cuja versão para investigação foi elaborada por Machado, Gonçalves e Matos (2000) e o Sexual Experiences Survey - Short Form Perpetration (SES-SFP) (Koss, Bachar \& SES Collaborative, 2004, versão traduzida por Peixoto, J. \& Machado, C., 2006). 
O EVC é composto por 29 itens, relativos a crenças socioculturais comuns sobre a violação, face aos quais os sujeitos se devem posicionar em termos de grau de concordância/discordância. Os itens estão construídos segundo a concepção de que as vítimas serão do sexo feminino e os seus perpetradores do sexo masculino. As opções de resposta encontram-se formuladas numa escala do tipo Likert de 5 pontos (em que 1 corresponde a "discordo totalmente" e 5 a "concordo totalmente"). A sua nota total mede o grau de legitimação/tolerância face à violência sexual sobre as mulheres. Os 29 itens da escala correlacionam-se fortemente com o seu total (as correlações variam entre um mínimo de .41 e um máximo de .76). A Análise de Componentes Principais, seguida de rotação Varimax, permitiu obter três fatores que, no seu conjunto, explicam $53.5 \%$ da variância da escala. O primeiro fator ["legitimação da violência pelo consentimento feminino"] explica $43.8 \%$, o segundo ["legitimação da violência através do comportamento sexual prévio da mulher"] explica 5.2\% e o terceiro ["legitimação da violação pela normalidade do ofensor"] explica $4.5 \%$ da variância. A consistência interna da ECV, obtida através do coeficiente alpha de Cronbach, é de .93 (Machado, Gonçalves, \& Matos, 2000).

Para determinar as taxas de prevalência da perpetração de diferentes tipos de atos de violência sexual, utilizámos o SES-SFP (2004), cuja tradução e adaptação foram efetuadas por Peixoto e Machado (2006). A versão de 2004 avalia os comportamentos sexuais abusivos, perpetrados tanto por homens, como por mulheres. Para os propósitos deste estudo, considerámos pertinente adaptar os itens do questionário aos perpetradores masculinos. Assim, trata-se de um questionário de autorrelato constituído por seis itens, cada um com cinco opções de resposta relativas a estratégias de violência sexual perpetradas. Esta versão revista para "perpetradores" (Koss \& Dinero, 1988; Koss \& Gidycz, 1985; Koss \& Oros, 1982) encontra-se dividida em duas partes, uma dedicada às experiências sexuais abusivas ocorridas no último ano e outra às experiências sexuais abusivas ocorridas desde os 14 anos. Mais concretamente, é pedido aos participantes para estimar o número de vezes que adotaram cada uma das estratégias sexualmente abusivas, em cada um dos seis itens, no último ano e desde os 14 anos, numa escala de frequência entre "0 vezes" e "3 ou mais vezes". O SES permite ainda colocar os participantes em categorias mutuamente exclusivas, de acordo com o tipo de atos sexuais mais severos praticados ("não perpetrador"; "perpetrador de coerção sexual"; "perpetrador de tentativa de violação" e "violador"), assim como determinar a frequência dos atos sexuais abusivos praticados pelos participantes categorizados como perpetradores (frequência da coerção sexual entre os "perpetradores de coerção sexual"; frequência da tentativa de violação entre os "perpetradores de tentativa de violação" e frequência da violação entre os "violadores"). O questionário apresenta uma consistência interna de 0.70 (Koss et al., 2004). 


\section{Procedimentos}

A fase de entrega e recolha dos questionários utilizados neste estudo compreendeu dois momentos distintos. Num primeiro momento, após obtida a autorização do Departamento de Desporto e Cultura de uma Universidade do Norte do País e dos técnicos responsáveis por cada uma das modalidades desportivas participantes nesta investigação, procedeu-se à entrega e recolha dos questionários junto do grupo dos atletas nos treinos semanais das respetivas equipas. Num segundo momento, após identificadas as variáveis sociodemográficas (idade) e formativas (ano e curso de formação) presentes no grupo dos "atletas", pedimos autorização aos diretores dos cursos dos não atletas participantes e procedemos à administração dos questionários nos tempos letivos cedidos pelos docentes.

A participação foi, em todos os casos, voluntária. Os participantes dos dois grupos foram, previamente, informados acerca do objetivo do estudo a realizar e explicitou-se a importância da sua contribuição para o mesmo. A confidencialidade e anonimato dos participantes foram assegurados através da garantia de uso exclusivo dos dados recolhidos para o presente estudo e da não recolha de dados de identificação pessoal.

\section{Análise estatística dos dados}

De acordo com os objetivos de investigação e as variáveis em estudo, os dados recolhidos foram submetidos a uma análise estatística através do programa informático IBM SPSS, versão 20.0, com o qual se efetuaram análises descritivas e inferenciais. Tendo-se verificado que os pressupostos subjacentes à utilização de testes paramétricos não estavam cumpridos, recorremos ao teste não paramétrico Mann-Whitney para amostras independentes, de modo a explorar a existência de diferenças entre os dois grupos de estudantes universitários do sexo masculino, atletas e não atletas, em termos do grau global de legitimação/tolerância e da tipologia de crenças face à violência sexual no namoro e da frequência de atos de violência sexual na intimidade. Utilizámos ainda o teste do Qui-quadrado para determinar a associação entre perpetradores e tipo de atos sexuais abusivos mais severos perpetrados.

\section{Resultados}

\section{Crenças socioculturais face à violência sexual no namoro}

A análise global das crenças dos participantes sobre a violência sexual no namoro revelou que ambos os grupos, atletas e não atletas, evidenciavam níveis reduzidos de concordância face aos mitos socioculturais apresentados. 
As respostas dadas à ECV pelo grupo dos atletas apresentaram um nível médio de $59.0(D P=15.26)$, enquanto que as respostas do grupo de não atletas registaram uma média de $61.2(D P=22.18)$. Estes dados indicaram uma tendência de resposta no sentido do "discordo" por parte dos dois grupos em relação à generalidade dos itens que compõem a escala. Relativamente a cada um dos fatores que integram o instrumento: (F1) Legitimação da violação pelo consentimento feminino (atletas $-M=33.9, D P=9.53$; não atletas $-M=34.6, D P=13.24$ ); (F2) Legitimação da violação pelo comportamento prévio da mulher (atletas $-M=27.74, D P=8.59$; não atletas $-M$ $=31.5, D P=13.36)$ e (F3) Legitimação da violação pela normalidade do ofensor (atletas $-M=14.1, D P=3.32$, não atletas $-M=14.4, D P=5.53$ ), todos revelaram uma tendência de resposta no sentido da baixa legitimação da violência sexual.

A análise das diferenças da ordem média entre os dois grupos, realizada através do Teste de Mann-Whitney para amostras independentes, indicou que não há diferenças estatisticamente significativas entre atletas e não atletas em relação ao nível global de legitimação da violência sexual, $U=1231$, $p=, 896$. Quanto aos fatores da escala, verificou-se igualmente a ausência de diferenças significativas entre os grupos no que concerne à legitimação da violação pelo consentimento feminino, (F1) $U=1206,50, p=, 764$, à legitimação da violação pelo comportamento sexual prévio da mulher, (F2) $U=1088, p=, 264]$; e à legitimação da violação pela normalidade do ofensor, (F3) $U=1249,50, p=, 997$ (cf. Tabela 1).

Tabela 1. Diferenças entre as médias dos grupos de atletas e não atletas em termos globais e dos fatores de legitimação face à violência sexual

\begin{tabular}{|c|c|c|c|c|c|}
\hline \multirow{2}{*}{$\begin{array}{c}\text { Escala de Crenças sobre a } \\
\text { Violação }\end{array}$} & \multicolumn{2}{|c|}{$\begin{array}{l}\text { Atletas } \\
(n=50)\end{array}$} & \multicolumn{2}{|c|}{$\begin{array}{l}\text { Não Atletas } \\
\quad(n=50)\end{array}$} & \multirow[b]{2}{*}{$U$} \\
\hline & Média & DP & Média & DP & \\
\hline ECV Total & 59,0 & 15,26 & 61,2 & 22,18 & 1231 \\
\hline $\begin{array}{l}\text { F1 - Legitimação da violação } \\
\text { pelo consentimento feminino }\end{array}$ & 33.9 & 9,53 & 34,6 & 13,24 & 1206,50 \\
\hline $\begin{array}{l}\text { F2 - Legitimação da violação } \\
\text { pelo comportamento sexual } \\
\text { prévio da mulher }\end{array}$ & 27,7 & 8,59 & 31,5 & 13,36 & 1088 \\
\hline $\begin{array}{l}\text { F3 - Legitimação da violação } \\
\text { pela normalidade do ofensor }\end{array}$ & 14,1 & 3,32 & 14,4 & 5,53 & 1249,50 \\
\hline
\end{tabular}


Prevalência da perpetração de diferentes tipos de comportamentos de violência sexual nas relações de namoro

A análise da categorização dos atletas e não atletas de acordo com a tipologia de atos sexuais mais abusivos perpetrados, tanto nas relações passadas, como nas relações atuais, revelou que apesar de uma percentagem elevada de atletas (passado - 58\%; presente - 68\%) e não atletas (passado $60 \%$; presente - 64\%) se assumirem como "não perpetradores", há uma percentagem preocupante de atletas (passado - 26\%; presente $-22 \%$ ) e de não atletas (passado - 24\%; presente $-26 \%$ ), categorizados em termos da perpetração da "forma mais grave" de agressão sexual - "violação". Importa ainda referir que a perpetração de atos sexuais "menos graves" esteve igualmente presente nas vivências relacionais íntimas destes grupos, embora com uma expressão menos significativa (e.g., coerção sexual) (cf. Tabela 2). Ainda a este nível, verificou-se que não há uma associação significativa entre o tipo de grupo (atletas vs não atletas) e a tipologia de atos de violência sexual perpetrados, tanto no passado $\left(\chi^{2}(3)=, 348, p=, 951\right)$, como no presente $\left(\chi^{2}\right.$ $(3)=, 703, p=, 872)$.

Tabela 2. Prevalência da perpetração de atos sexualmente abusivos nas relações amorosas passadas (desde os 14 anos) e nas relações amorosas atuais (último ano) nos grupos dos atletas e não atletas

\begin{tabular}{lcccc}
\hline Tipologia de ofensores sexuais & \multicolumn{2}{c}{ Atletas } & \multicolumn{2}{c}{ Não Atletas } \\
\hline & $\begin{array}{c}\text { Desde os } \\
14 \text { anos }\end{array}$ & Último ano & $\begin{array}{c}\text { Desde os } \\
14 \text { anos }\end{array}$ & Último ano \\
\cline { 2 - 5 } & $\%(n)$ & $\%(n)$ & $\%(n)$ & $\%(n)$ \\
\hline Não perpetradores & $58(29)$ & $68(34)$ & $60(30)$ & $64(32)$ \\
$\begin{array}{l}\text { Perpetradores de coerção } \\
\text { sexual }\end{array}$ & $10(5)$ & $6(3)$ & $12(6)$ & $8(4)$ \\
$\begin{array}{l}\text { Perpetradores de tentativa de } \\
\text { violação }\end{array}$ & $6(3)$ & $4(2)$ & $4(2)$ & $2(1)$ \\
Perpetradores de violação & $26(13)$ & $22(11)$ & $24(12)$ & $26(13)$ \\
\hline
\end{tabular}

A análise comparativa das respostas dadas ao SES-SFP pelo grupo de atletas, com as respostas dadas pelo grupo de não atletas, ao nível da frequência de perpetração de atos sexualmente abusivos, quer nas relações amorosas passadas, quer nas atuais, sugeriu que nenhum dos dois grupos 
apresentava uma frequência média de perpetração de atos de agressão sexual significativamente superior. Não obstante, observou-se, tanto no grupo de atletas como no de não atletas, uma frequência média mais elevada na perpetração de atos de violência sexual mais graves e intrusivos, designadamente a "violação", tanto no passado como no presente, comparativamente com uma menor prevalência de formas menos graves de violência sexual (e.g., coerção sexual) (cf. Tabela 3).

Tabela 3. Frequência média da perpetração de atos sexualmente abusivos no passado (desde os 14 anos) e no presente (último ano) nos grupos dos atletas e não atletas

\begin{tabular}{cccccccccc}
\hline SES-SFP & \multicolumn{4}{c}{ Atletas } & \multicolumn{4}{c}{ Não atletas } \\
& \multicolumn{3}{c}{ Passado } & Presente & Passado & \multicolumn{2}{c}{ Presente } \\
\cline { 2 - 9 } & M & DP & M & DP & M & DP & M & DP \\
\hline Coerção sexual & 0,7 & 2,22 & 0,2 & 1,10 & 0,5 & 1,6 & 0,4 & 1,54 \\
Tentativa de violação & 0,4 & 2,10 & 0,3 & 1,99 & 0,8 & 4,4 & 0,6 & 4,24 \\
Violação & 1,4 & 3,6 & 1,1 & 3,33 & 2,6 & 6,0 & 2,3 & 5,43 \\
\hline
\end{tabular}

\section{Discussão}

A análise dos dados recolhidos não suporta a hipótese empírica de que os atletas apresentam níveis superiores de legitimação/tolerância face à violência sexual na intimidade e uma taxa mais elevada de prevalência de comportamentos sexualmente abusivos, quando comparados com os não atletas.

$\mathrm{Na}$ globalidade, os dois grupos, participantes neste estudo, demonstram, ao nível cognitivo-atitudinal, um reduzido grau de suporte face às crenças socioculturais comummente utilizadas para justificar ou desculpabilizar a violência sexual perpetrada pelo homem sobre a mulher. Concomitantemente, atletas e não atletas não evidenciam diferenças estatisticamente significativas quer ao nível do grau global de legitimação/tolerância face à violência sexual na intimidade, quer no que se refere à tipologia de crenças face à violência sexual.

Em termos comportamentais, verifica-se que ambos os grupos apresentam resultados similares, evidenciando uma taxa de perpetração de atos sexuais abusivos na intimidade superior no "tipo mais grave" de violência sexual - a violação -, tanto no presente (último ano) como no passado (desde os 14 anos). 
Assim, estes resultados parecem corroborar a ideia veiculada por alguns autores (e.g., Smith \& Stewart, 2003) de que a conceptualização da cultura atlética como facilitadora da violência sexual contra as mulheres na intimidade pode não passar de uma "sobressimplificação", motivada pelo estatuto e visibilidade social dos atletas entre a população universitária. Adicionalmente, há estudos (e.g., Sawyer, Thompson \& Chicorelli, 2002) que indicam que os atletas não são um grupo homogéneo e que, por conseguinte, só fará sentido estudar o papel da afiliação atlética nas crenças e comportamentos dos homens atletas, se se tiver em consideração a forma como essa variável se relaciona ou potencia a influência moderadora de outras variáveis (e.g., vitimação na infância, consumo de álcool, ideologia de masculinidade, características de personalidade).

O reduzido corpo de estudos que se conhece sobre o tema em estudo sugere, contrariamente aos presentes resultados, a existência de uma associação significativa entre a participação atlética em modalidades agressivas e competitivas de contacto (e.g., Smith \& Stewart, 2003), o maior grau de adesão a mitos legitimadores da violência sexual (e.g., Boeringer, 1999) e a perpetração frequente de agressões sexuais na intimidade (e.g., Frintner \& Rubinson, 1993).

Uma das possíveis alternativas para a explicação dos resultados obtidos, diz respeito ao efeito da desejabilidade social associada aos questionários de autorrelato. Neste sentido, a literatura aponta para que as medidas de autorrelato possam desencadear nos participantes a tendência para responder de acordo com o desejado socialmente, sobretudo no que concerne às crenças face a problemáticas tão sensíveis, no plano social, como a violência sexual sobre as mulheres. Não obstante, ao nível comportamental, parece-nos que os resultados obtidos podem sugerir, por um lado, a tentativa dos participantes em agir de acordo como os ideais socioculturais de masculinidade, assumindo o relato de comportamentos de violência sexual como uma demonstração de controlo, poder e autoridade sobre as mulheres e, por outro, a oportunidade de admissão anónima e confidencial da prática de atos sexuais violentos nas suas relações amorosas.

Outro aspeto particularmente relevante prende-se com as diferenças existentes entre a cultura atlética no campus universitário norte-americano e a cultura inerente à prática desportiva nas universidades portuguesas, que podem desempenhar um papel preponderante na explicação da discrepância entre os resultados do presente estudo e os dados empíricos dos estudos norte-americanos sobre o tema. Assim, os níveis elevados de competitividade e agressividade que caracterizam a cultura atlética norte-americana (Davis, 2000), podem ajudar a explicar os elevados indicadores de legitimação e perpetração de atos de violência sexual identificados nas investigações realizadas com atletas universitários norte-americanos. De facto, Eskenazi (1990, 
citado por McMahon, 2004) postula que a forma como o desporto é organizado (i.e., a cultura atlética) influencia o desenvolvimento de uma masculinidade hostil face às mulheres, não sendo a participação desportiva per se que torna os atletas mais propensos a legitimar e/ou cometer atos de violência sexual na intimidade.

Deste modo, torna-se importante analisar, clarificar e aprofundar, em futuras investigações, a relação entre cultura atlética, masculinidade e violência sexual contra as mulheres na intimidade (McMahon, 2004, 2007).

\section{Limitações}

Este estudo apresenta algumas limitações que devem ser tidas em consideração na interpretação dos resultados obtidos. Uma primeira limitação prende-se, desde logo, com o método de amostragem. Foi utilizada uma amostra reduzida e de conveniência, recolhida num único campus universitário, o que à semelhança do que sucede com a grande maioria dos estudos realizados nesta área, não permite extrair generalizações para toda a população universitária atlética portuguesa

Por outro lado, o efeito da desejabilidade social associado aos instrumentos de autorrelato administrados poderá também ter influenciado os resultados obtidos, no sentido quer da reduzida legitimação face à violência sexual, quer do sobrerrelato da perpetração de comportamento sexualmente abusivos na intimidade.

Por último, o facto de se tratar de um estudo meramente quantitativo, com recurso exclusivo a questionários de autorrelato, não permite compreender algumas questões fenomenológicas relacionadas, por exemplo, com as circunstâncias em que as condutas sexuais abusivas ocorrem, os motivos que lhes são atribuídos, a sequência interativa dos atos sexuais abusivos, nem a significação atribuída à agressão pelos seus intervenientes.

\section{Conclusões}

Os resultados obtidos não corroboram os dados empíricos da maioria das investigações internacionais realizados em torno deste tema, sendo indicativos de presença de crenças globalmente adequadas. Não obstante, ao nível comportamental, constatámos a existência de uma percentagem significativa de atletas e não atletas que se inserem na categoria de "violadores". Este dado alerta, assim, para que a violência sexual, na sua expressão mais severa, possa ser uma realidade prevalente entre a população universitária estudada. 
No que se refere a investigações futuras sobre esta temática, consideramos importante a expansão de estudos nacionais sobre a problemática da violência sexual na intimidade, junto de uma amostra representativa da população atlética portuguesa, de modo a explorar e compreender melhor as complexidades da hipotética relação entre participação desportiva, masculinidade e violência sexual. Algumas sugestões a este nível prendem-se com a relevância empírica de se englobar atletas de outras universidades e utilizar uma metodologia complementar de cariz qualitativo, para uma análise mais detalhada das crenças e comportamentos sexuais abusivos dos atletas. Ao nível das crenças, poderá ser também interessante analisar se os universitários que admitem cometer actos sexuais abusivos sustentam crenças diferentes dos não perpetradores.

Em suma, este estudo poderá constituir-se como um pequeno no sentido de abrir caminho a futuras investigações sobre esta temática e, desta forma, contribuir para a prossecução do objetivo a que nos propusemos inicialmente de compreender e refletir acerca da relação entre a participação atlética masculina e a violência sexual contra as mulheres.

\section{Referências}

Abbey, A., McAuslan, P., Zawacki, T., Clinton, A. M., \& Buck, P. O. (2001). Attitudinal, experiential and situational predictors of sexual assault perpetration. Journal of Interpersonal Violence, 16, 784-807.

Banyard, V. L., Plante, E. G., \& Moynihon, M. M. (2004). Bystander education: Bringing a broader community perspective to sexual violence prevention. Journal of Community Psychology, 32, 61-79.

Berkowitz, A. (1992). College men as perpetrators of acquaintance rape and sexual assault: A review of recent research. Journal of American College Health, 40, 175-181.

Boeringer, S. (1999). Associations of rape-supportive attitudes with fraternal and athletic participation. Violence Against Women, 5, 81-90.

Brownmiller, S. (1975). Against our will: Men, women, rape. New York: Bantam.

Burt, M. (1980). Cultural myths and supports for rape. Journal of Personality and Social Psychology, 38, 217-230.

Caridade, S. \& Machado, C. (2008). Violência sexual no namoro: A relevância da prevenção. Psicologia, Vol. 22, 77 - 104.

Caridade, S. \& Machado, C. (2010). Violência na intimidade juvenil: prevalência, factores de risco e atitudes. In C. Machado (coord.). "Novas" formas de vitimação criminal (pp. 13-59). Braga: Psiquilíbrios Edições.

Carr, J. L., \& Van Deusen, K. M. (2004). Risk factors for male sexual aggression on college campuses. Journal of Family Violence, 19, 279-289.

Castro, A. J., Cruz, O., \& Martins, S. (2005). Programa de formação e prevenção (primária e secundária) da violência nas relações de namoro: Bem me quer... 
Mal me quer. (Não publicado). Braga: Escola de Psicologia, Universidade do Minho.

Connell, R. W. (2005) Hegemonic masculinity - rethinking the concept. Gender \& Society, 19, 829-859.

Costa, J. M. B., \& Alves, L. B. (1999). Perspectivas teóricas e investigação do domínio da delinquência sexual em Portugal. Revista Portuguesa de Ciência Criminal, 9, 281-312.

Costa, I. R \& Sani, A. I. (no prelo). O Abuso e as Crenças sobre a Violência nas Relações Amorosas de Estudantes Universitários. Revista Lusófona de Ciências da Mente e do Comportamento, 8.

Crosset, T., Benedict, J., \& McDonald, M. (1995). Male student-athletes reported for sexual assault: A survey of campus police departments and judicial affairs offices. Journal of Sport and Social Issues, 19, 125-144.

Crosset. T., Ptacek, J., McDonald, \& M. Benedict, J. (1996). Male student-athletes and violence against women. Violence Against Womem, 2, 163-179.

Davis, T. (2000). Programming for men to reduce sexual violence. New Directions for Student Services, 90, 79-89.

Day, K. (1994). Assault prevention as social control: Women and fear of sexual assault on urban college campuses. Ann Arbor: UMI.

DeKeseredy, W. S., \& Schwartz, M. D., (1998). Male peer support and woman abuse in postsecondary school courtship suggestions for new directions in sociological research. In R. K. Bergen (Ed.), Issues in intimate violence (pp.83-96). Thousand Oaks: Sage Publications.

Feltey, K. M., Ainslie, J. J., \& Geib, A. (1991). Sexual coercion attitudes among high school students. The influence of gender and rape education. Youth \& Society, 23, 229-250.

Forbes, G. B., Adams-Curtis, L. E., Pakalka, A. H., \& White, K. B. (2006). Dating Aggression, Sexual Coercion, and Aggression-Supporting Attitudes Among College Men as a Function of Participation in Aggressive High School Sports. Violence Against Women, 12, 441-55.

Fritner, M. P., \& Rubinson, L. (1993). Acquaintance rape: The influence of alcohol, fraternity membership and sports team membership. Journal of Sex Education and therapy, 19, 272-284.

Geiger, B., Fischer, M., \& Eshet, Y. (2004). Date-rape-supporting and victim-blaming attitudes among high school students in a multiethnic society. Journal of Interpersonal Violence, 19, 406-426.

Glass, N., Fredland, N., Campbell, J., Yonas, M., Sharps, P., \& Kub, J. (2003). Adolescent dating violence: Prevalence, risk factors, health outcomes and implications for clinical pratice. Jognn Clinical Issues, 32, 227-238.

Gover, A. R. (2004). Risky lifestyles and dating violence: A theoretical test of violent victimization. Journal of Criminal Justice, 32, 171-180.

Gross, A., Winslett, A., Roberts, M., \& Grohn, C. (2006). An examination of sexual violence against college women. Violence Against Women, 12, 288-300.

Himelein, M. (1995). Risk factos for sexual victimization in dating. Psychology of Women Quartely, 19, 31-48. 
Humphrey, J. A. \& White, J. W. (2000). Women's vulnerability to sexual assault form adolescence to young adulthood. Journal of Adolescent Health, 27, 419-424 .

Jackson, S. M. (1999). Issues in the dating violence research: A review of the literature. Agression and Violent Behavior, 4, 233-247.

Jackson, T. L., \& Davis, J. L.(2000). Prevention of sexual and physical assault toward women: A program for male athletes. Journal of Community Psychology, 28, 589-605.

Koss, M. P., Bachar, K. J., \& SES Colaborative (2004). Sexual Experiences Survey Short Form Perpetration“ (SES-SFP), traduzido por Peixoto, J. \& Machado, C. (versão para investigação). Braga: Escola de Psicologia, Universidade do Minho

Koss, M. P. \& Cleveland, H. H. (1997). Stepping on toes: Social rotes of date rape lead to intractability and politicization. In M. D. Schwartz (Ed.). Researching sexual violence against women: Methodological and personal perspectives (4-21). Thousand Oaks: Sage Publications.

Koss, M. \& Dinero, T. (1989). Discriminant analysis of risk factors for sexual victimization among a national sample of college women. Journal of Consulting and Clinical Psychology, 57, 242-250.

Koss, M. P., Dinero, T. E., Seibel, C. A, \& Cox, S. L. (1988). Stranger and acquaintance rape. Are there differences in the victim's experience? Psychology of Women Quarterly, 12, 1-24.

Koss, M. P. \& Gaines, J. A. (1993). The Prediction of Sexual aggression by Alcohol Use, Athletic Participation, and Fraternity Affiliation. The Journal of Interpersonal Violence, 8, 94-10.

Koss, M. \& Gidycz, C. (1985). The Sexual Experiences Survey: reliability and validity. Journal of Consulting and Clinical Psychology, 53, 442-443.

Koss, M., Gidycz, C., \& Wisniewski, N. (1987). The scope of rape: Incidence and prevalence of sexual and victimization in a national sample of higher education studentws. Journal of Consulting and Clinical Psychology, 55, 162-170.

Koss, M. \& Oros, C. (1982). Sexual Experiences Survey: A research instrument investigating sexual aggression and victimization. Journal of Consulting and Clinical Psychology, 50, 455-457.

Lonsway, K. A. \& Fitzgerald, L. F. (1994). Rape myths: In review. Psychology of Women Quarterly, 18, 133-164.

Machado, C., Gonçalves, M., \& Matos, M. (2000). Escala de crenças sobre a violação (ECV). Braga: Escola de Psicologia, Universidade do Minho.

Machado, C., Matos, M., \& Moreira, A. I. (2003). Violência nas relações amorosas: Comportamentos e atitudes na população universitária. Psychologica, 33, 69-83 .

Mahoney, P., Williams, L. M., \& West, C. M. (2001). Violence against women by intimate relationships partners. In C. M. Renzetti, J. L. Edleson \& R. K. Bergen (Eds.). Sourcebook on violence against women (pp. 143-178). Thousand Oaks: Sage Publications. 
Malamuth, N. M., Sockloskie, R., Koss, M. P., \& Tanaka, J. (1991). The characteristics of agressors agnaist women: Testing a model using a national sample of college students. Journal of Consuling and Clinical Psychology, 59, 670-681.

Malamuth, N., Heavey, C., \& Linz, D. (1996). The confluence model of sexual aggression: Combining hostile masculinity and impersonal sex. Journal of Offender Rehabilitation, 23, 13-37.

Marlin, A. J. \& Russo, N. F. (1999). Feminist perspectives on male violence against women: Critiquing O'Neil and Harway's model. In M. Harway \& J. O'Neil (Eds), What causes men's violence against women? (pp. 18-35), Thousands Oaks: Sage Publications.

Martins, S. \& Machado, C. (2010). Violência sexual em jovens adultos: caracterização e implicações para a investigação. In C. Machado (coord.). "Novas" formas de vitimação criminal (pp. 61-120). Braga: Psiquilibrios Edições.

Matos, M., Caridade, S., Machado, C., \& Silva, M. J. (2006). Prevenção da violência nas relações de intimidade: Intervenção com jovens em contexto escolar. Psicologia: Teoria e Prática (Universidade Presbiteriana Mackenzie), 8, 55$-76$.

Marx, B. P., Wie, V. W. \& Gross, A. M. (1996). Date rape risk factors: A review and methodological critique of the literature. Aggression and Violent Behavior, $1,27-45$.

Maxwell, C. D., Rubinson, A. L. \& Post, L.A. (2003). The nature and predictors of sexual victimization and offending among adolescents. Journal of Youth and Adolescence, 32, 465-477.

McMahon, P. (2000). The public health approach to the prevention of sexual violence. Sexual Abuse: A Journal of Research and Treatment, 12, 27-26.

McMahon, S. (2004). Student-Athletes, Rape-Supportive Culture, and Social Change. Department of Sexual Assault Services and Crime Victim, Rutgers, State University of New York. Acedido em 4 de Novembro de 2011 em http://sexualassault.rutgers.edu

McMahon, S. (2007). Understanding community specific rape myths: Exploring student-athlete culture. Affilia, 22, 354-370.

Molidor, C., Tolman, R. M.,\& Kober, J. (2000). Gender and contextual factors inadolescent dating violence. Prevention Researcher, 7, 1-4.

Muhelenhard, C. L. \& Linton, M. A. (1987). Date Rape and Sexual Assault in Dating Situatons: Incidence and Risk Factors. Journal of Conseling Psychology, 34, 186-196.

Paiva, C. \& Figueiredo, B. (2004). Abuso no relacionamento íntimo: Estudo de prevalência em jovens adultos portugueses. Psychologica, 36, 75-107.

Price, E. L., Byers, E. S., \& Dating violence research team (1999). The attitudes towards dating violence scales: Development and initial validation. Journal of Family Violence, 4, 387-415.

Rozée, P. D. (1993). Forbidden or forgiven? Rape in cross-cultural perspective. Psychology of Women Quarterly, 17, 499-514.

Sanday, P. (1990). Fraternity gang rape: Sex, brotherhood, and privilege on campus. New York: New York University Press. 
Sawyer, R. G., Thompson, E. E., \& Chicorelli, A. M. (2002). Rape myth acceptance among intercollegiate student athletes: A preliminary examination. American Journal of Health Studies, 18, 19-25.

Schwartz, M. D. \& DeKeseredy, W. S. (1997). Sexual Assault on the college campus. The role of male peer support. Thousand Oaks: Sage Pulications.

Smith, D. \& Stewart, S. (2003). Sexual Agression and Sports Participation. Journal of Sport Behavior, 26, 384-395.

Söchting, I., Fairbrother, N. \& Koch, W. J. (2004). Sexual Assault of Women: Prevention efforts and risk factors. Violence Against Women, 10, 73-93.

Truman, D. M., Tokar, D. M., \& Fisher, A. R. (1996). Dimensions of masculinity: Relations to date rape supportive attitudes and sexual aggression in dating situations. Journal of Counseling and Development, 74, 555-562.

Yeater, E. A. \& O’ Donohue, W. (1999). Sexual assault prevention programs: Current issues, future directions and the potential efficacy of interventions with women. Clinical Psychology Review, 19, 739-771. 